\title{
Gauge Boson Mixing in the 3-3-1 Models with Discrete Symmetries
}

\author{
P. V. Dong, V. T. N. Huyen, H. N. Long, and H. V. Thuy \\ Institute of Physics, VAST, 10 Dao Tan, Ba Dinh, Hanoi 1000, Vietnam \\ Correspondence should be addressed to P. V. Dong, pvdong@iop.vast.ac.vn
}

Received 19 September 2011; Accepted 6 December 2011

Academic Editor: Marc Sher

Copyright (C) 2012 P. V. Dong et al. This is an open access article distributed under the Creative Commons Attribution License, which permits unrestricted use, distribution, and reproduction in any medium, provided the original work is properly cited.

The mixing among gauge bosons in the 3-3-1 models with the discrete symmetries is investigated. To get tribimaximal neutrino mixing, we have to introduce sextets containing neutral scalar components with lepton number $L=1,2$. Assignation of VEVs to these fields leads to the mixing of the new gauge bosons and those in the standard model. The mixing in the charged gauge bosons leads to the lepton number violating interactions of the $W$ boson. The same situation happens in the neutral gauge boson sector.

\section{Introduction}

The experimental evidences of nonzero neutrino masses and mixing [1] have shown that the standard model of fundamental particles and interactions must be extended. Among many extensions of the standard model known today, the models based on gauge symmetry $\mathrm{SU}(3)_{C} \otimes \mathrm{SU}(3)_{L} \otimes \mathrm{U}(1)_{X}$ (called 3-3-1 models) [2-9] have interesting features. First, $\left[\mathrm{SU}(3)_{L}\right]^{3}$ anomaly cancelation requires that the number of $S U(3)_{L}$ fermion triplets must be equal to that of antitriplets. If these multiplets are respectively enlarged from those of the standard model, the fermion family number is deduced to be a multiple of the fundamental color number, which is three, coinciding with the observation (see Frampton in [2]). In addition, one family of quarks has to transform under $\mathrm{SU}(3)_{L}$ differently from the other two. This can lead to an explanation why the top quark is characteristically heavy (see, e.g., [10]). To complete the fundamental representations for leptons, the right-handed neutrinos or neutral fermions can be imposed which imply natural seesaw mechanisms for the neutrino small masses [11]. The 3-3-1 models can also provide a solution of electric charge quantization observed in the nature [12-16]. 
Table 1: Character table of $S_{3}$, where $\chi$ stands for character of representation and $C$ for class.

\begin{tabular}{lllccc}
\hline Class & $n$ & $h$ & $\chi_{\underline{1}}$ & $\chi_{\underline{1^{\prime}}}$ & $\chi_{\underline{2}}$ \\
\hline$C_{1}$ & 1 & 1 & 1 & 1 & 2 \\
$C_{2}$ & 2 & 3 & 1 & 1 & -1 \\
$C_{3}$ & 3 & 2 & 1 & -1 & 0 \\
\hline
\end{tabular}

There are two typical versions of the 3-3-1 models concerning respective lepton contents. In the minimal 3-3-1 model [2-4] the lepton triplets include ordinary leptons of the standard model such as $\left(v_{L}, l_{L}, l_{R}^{c}\right)$. The 3-3-1 model with right-handed neutrinos [5-9] introduces right-handed neutrinos into the lepton sector, that is, $\left(v_{L}, l_{L}, v_{R}^{c}\right)$ and $l_{R}$. In the framework of 3-3-1 models, to explain the smallness of neutrino masses and the tribimaximal mixing [17-20]

$$
U^{\mathrm{HPS}}=\left(\begin{array}{ccc}
\frac{2}{\sqrt{6}} & \frac{1}{\sqrt{3}} & 0 \\
-\frac{1}{\sqrt{6}} & \frac{1}{\sqrt{3}} & \frac{1}{\sqrt{2}} \\
-\frac{1}{\sqrt{6}} & \frac{1}{\sqrt{3}} & -\frac{1}{\sqrt{2}}
\end{array}\right)
$$

we should propose another variant of the lepton sector such as $\left(v_{L}, l_{L}, N_{R}^{c}\right)$ and $l_{R}$ where $N_{R}$ are neutral chiral fermions carrying no lepton number (called 3-3-1 model with neutral fermions), and including discrete symmetries either $A_{4}$ or $S_{4}$ [21, 22]. The 3-3-1 model with neutral fermions based on $S_{3}$ flavor symmetry instead of $A_{4}, S_{4}$ has been studied in [23].

One of the most important ingredients is the sextets in which neutral scalar fields carrying lepton number $L=1$ or 2 . Assignation of VEVs to these fields leads to the mixing among new gauge bosons and that of the SM similarly in the economical 3-3-1 model [24$26]$, and such mixing leads to the lepton violating interactions. In this work we will pay attention to gauge bosons in the mentioned 3-3-1 models and give some phenomenological consequences.

The rest of this work is follows. In Section 2 we give a review of the 3-3-1 model with neutral fermions-based $S_{3}$ flavor symmetry. The other models with $A_{4}$ and $S_{4}$ can be done similarly, thus should be skip. Section 3 identifies gauge bosons and obtained the mixings among the standard model gauge bosons and the new ones. Section 4 is devoted to charged currents and give a constraint on the charged gauge boson mixing-angle. Finally we make conclusions in Section 5.

\section{Brief Review of the Model}

Before looking into the model, we provide a sketch of $S_{3}$ group theory $[27,28]$. The $S_{3}$ that is a permutation group of three objects has six elements divided into three conjugacy classes. It possesses three nonequivalent irreducible representations $\underline{1}, \underline{1}$ ' of one dimension, and $\underline{2}$ of two dimensions. Denoting $n$ and $h$ as the order of class and the order of elements within each class, respectively, the character table is given by Table 1 . 
We will work in the basis that $\underline{2}$ is complex (see, e.g. [27]). Decomposition rules are

$$
\begin{aligned}
& \underline{1} \otimes \underline{1}=\underline{1}(11), \quad \underline{1}^{\prime} \otimes \underline{1}^{\prime}=\underline{1}(11), \quad \underline{1} \otimes \underline{1}^{\prime}=\underline{1}^{\prime}(11), \\
& \underline{1} \otimes \underline{2}=\underline{2}(11,12), \quad \underline{1}^{\prime} \otimes \underline{2}=\underline{2}(11,-12), \\
& \underline{2} \otimes \underline{2}=\underline{1}(12+21) \oplus \underline{1^{\prime}}(12-21) \oplus \underline{2}(22,11) .
\end{aligned}
$$

Here the first and second factors of the terms appearing in the parentheses indicate to the multiplet components of the first and second representations given in 1.h.s, respectively. In this basis, the conjugation rules are given by

$$
\underline{2}^{*}\left(1^{*}, 2^{*}\right)=\underline{2}\left(2^{*}, 1^{*}\right), \quad \underline{1}^{*}\left(1^{*}\right)=\underline{1}\left(1^{*}\right), \quad \underline{1}^{\prime *}\left(1^{*}\right)=\underline{1}^{\prime}\left(1^{*}\right) .
$$

The lepton number in the 3-3-1 model with $S_{3}$ symmetry [23] does not commute with the gauge symmetry. It is thus better to work with a new lepton charge $\mathcal{L}$ related to the lepton number $L$ by diagonal matrices $L=x T_{3}+y T_{8}+\mathcal{L}$. Applying $L$ to the lepton triplet with the notation that $L\left(N_{R}\right)=0$, the coefficients are defined as $x=0, y=2 / \sqrt{3}$, and thus $L=(2 / \sqrt{3}) T_{8}+\mathcal{\perp}[29]$. The leptons and quarks under $\left[\mathrm{SU}(3)_{L}, U(1)_{X}, U(1)_{\mathcal{L}}, \underline{S}_{3}\right]$ symmetries correspondingly transform as follows:

$$
\begin{gathered}
\psi_{1 L}=\left(v_{1 L}, l_{1 L}, N_{1 R}^{c}\right)^{T} \sim\left[3,-\frac{1}{3}, \frac{2}{3}, \underline{1}\right], \quad l_{1 R} \sim[1,-1,1, \underline{1}], \\
\psi_{\alpha L}=\left(v_{\alpha L}, l_{\alpha L}, N_{\alpha R}^{c}\right)^{T} \sim\left[3,-\frac{1}{3}, \frac{2}{3}, \underline{2}\right], \quad l_{\alpha R} \sim[1,-1,1, \underline{2}], \\
Q_{1 L}=\left(u_{1 L}, d_{1 L}, U_{L}\right)^{T} \sim\left[3, \frac{1}{3},-\frac{1}{3}, \underline{1}\right], \\
u_{1 R} \sim\left[1, \frac{2}{3}, 0, \underline{1}\right], \quad d_{1 R} \sim\left[1,-\frac{1}{3}, 0, \underline{1}\right], \quad U_{R} \sim\left[1, \frac{2}{3},-1, \underline{1}\right], \\
Q_{\alpha L}=\left(d_{\alpha L},-u_{\alpha L}, D_{\alpha L}\right)^{T} \sim\left[3^{*}, 0, \frac{1}{3}, \underline{2}\right], \\
u_{\alpha R} \sim\left[1, \frac{2}{3}, 0, \underline{2}\right], \quad d_{\alpha R} \sim\left[1,-\frac{1}{3}, 0, \underline{2}\right], \quad D_{\alpha R} \sim\left[1,-\frac{1}{3}, 1, \underline{2}\right],
\end{gathered}
$$

where $\alpha=2,3$ is a family index of the last two lepton and quark families, which are in order defined as the components of $\underline{2}$ representations.

To generate masses for the charged leptons, we need two scalar multiplets:

$$
\phi=\left(\begin{array}{c}
\phi_{1}^{+} \\
\phi_{2}^{0} \\
\phi_{3}^{+}
\end{array}\right) \sim\left[3, \frac{2}{3},-\frac{1}{3}, \underline{1}\right], \quad \phi^{\prime}=\left(\begin{array}{c}
\phi_{1}^{\prime+} \\
\phi_{2}^{\prime 0} \\
\phi_{3}^{\prime+}
\end{array}\right) \sim\left[3, \frac{2}{3},-\frac{1}{3}, \underline{1}^{\prime}\right]
$$


with VEVs $\langle\phi\rangle=(0, v, 0)^{T}$ and $\left\langle\phi^{\prime}\right\rangle=\left(0, v^{\prime}, 0\right)^{T}$. To generate masses for quarks, we additionally acquire the following scalar multiplets:

$$
\begin{aligned}
& x=\left(x_{1}^{0}, x_{2}^{-}, x_{3}^{0}\right)^{T} \sim\left[3,-\frac{1}{3}, \frac{2}{3}, \underline{1}\right], \\
& \eta=\left(\eta_{1}^{0}, \eta_{2}^{-}, \eta_{3}^{0}\right)^{T} \sim\left[3,-\frac{1}{3},-\frac{1}{3}, \underline{1}\right], \\
& \eta^{\prime}=\left(\eta_{1}^{\prime 0}, \eta_{2}^{\prime}, \eta_{3}^{\prime 0}\right)^{T} \sim\left[3,-\frac{1}{3},-\frac{1}{3}, \underline{1}^{\prime}\right] .
\end{aligned}
$$

Suppose that the VEVs of $\eta, \eta^{\prime}$, and $x$ are $u, u^{\prime}$ and $w$, where $u=\left\langle\eta_{1}^{0}\right\rangle, u^{\prime}=\left\langle\eta_{1}^{\prime 0}\right\rangle, w=\left\langle x_{3}^{0}\right\rangle$, and $\left\langle\eta_{3}^{0}\right\rangle,\left\langle\eta_{3}^{\prime 0}\right\rangle$, and $\left\langle x_{1}^{0}\right\rangle$ vanish. The exotic quarks get masses $m_{U}=f_{1} w$ and $m_{D_{1,2}}=f w$. In addition, $w$ has to be much larger than those of $\phi$ and $\eta$. Notice that the numbered subscripts are the indices of $\mathrm{SU}(3)_{L}$.

Because of the $\mathcal{L}$-symmetry, the couplings $\bar{\psi}_{L}^{c} \psi_{L} \phi$ and $\bar{\psi}_{L}^{c} \psi_{L} \phi^{\prime}$ are suppressed. We therefore propose a new $\mathrm{SU}(3)_{L}$ antisextet instead coupling to $\bar{\psi}_{L}^{c} \psi_{L}$ responsible for neutrino masses. The antisextet transforms as

$$
s=\left(\begin{array}{ccc}
s_{11}^{0} & s_{12}^{+} & s_{13}^{0} \\
s_{12}^{+} & s_{22}^{++} & s_{23}^{+} \\
s_{13}^{0} & s_{23}^{+} & s_{33}^{0}
\end{array}\right) \sim\left[6^{*}, \frac{2}{3},-\frac{4}{3}, \underline{2}\right]
$$

where the numbered subscripts are the $\mathrm{SU}(3)_{L}$ indices. Henceforth the indices of $S_{3}$ on scalar fields will be kept and should be understood. The VEVs of $s$ is set as $\left(\left\langle s_{1}\right\rangle,\left\langle s_{2}\right\rangle\right)$ under $S_{3}$, where

$$
\left\langle s_{i}\right\rangle=\left(\begin{array}{ccc}
\lambda_{i} & 0 & v_{i} \\
0 & 0 & 0 \\
v_{i} & 0 & \Lambda_{i}
\end{array}\right) \quad(i=1,2) .
$$

Due to the $S_{3}$ symmetry, all these VEVs are equal to each others, that is, $\lambda_{1}=\lambda_{2}, v_{1}=v_{2}$ and $\Lambda_{1}=\Lambda_{2}$, which can be found from the potential minimization.

With the scalar multiplets as defined, the Yukawa lagrangian is given by

$$
\begin{aligned}
\varrho_{Y}= & h_{1} \bar{\psi}_{1 L} \phi l_{1 R}+h\left(\bar{\psi}_{2 L} l_{2 R}+\bar{\psi}_{3 L} l_{3 R}\right) \phi+h^{\prime}\left(\bar{\psi}_{3 L} l_{3 R}-\bar{\psi}_{2 L} l_{2 R}\right) \phi^{\prime} \\
& +f_{1} \bar{Q}_{1 L} \chi U_{R}+f \bar{Q}_{L} x^{*} D_{R}+h_{1}^{u} \bar{Q}_{1 L} \eta u_{1 R}+h^{d} \bar{Q}_{L} \eta^{*} d_{R} \\
& +h^{\prime} d \bar{Q}_{L} \eta^{\prime *} d_{R}+h_{1}^{d} \bar{Q}_{1 L} \phi d_{1 R}+h^{u} \bar{Q}_{L} \phi^{*} u_{R}+h^{\prime} u \bar{Q}_{L} \phi^{\prime *} u_{R} \\
& +\frac{1}{2} x\left(\bar{\psi}_{2 L}^{c} \psi_{2 L} s_{1}+\bar{\psi}_{3 L}^{c} \psi_{3 L} s_{2}\right)+\frac{1}{2} y \bar{\psi}_{1 L}^{c}\left(\psi_{2 L} s_{2}+\psi_{3 L} s_{1}\right) \\
& + \text { H.c. }
\end{aligned}
$$


It is easily shown that the charged leptons and ordinary quarks get consistent masses [23]. However, this case does not lead to neutrino masses and mixing consistent with the experimental data. The analysis in $[21,22]$ shows that (i) a "perturbation" is required:

$$
\lambda_{1} \simeq \lambda_{2}, \quad v_{1} \simeq v_{2}, \quad \Lambda_{1} \simeq \Lambda_{2} .
$$

A possibility to derive this is to impose another antisextet $s^{\prime}$ but with the VEVs being very smaller than those of $s$, respectively. Thus, in the followings the $s^{\prime}$ should be skipped since it does not contribute at the first order. Otherwise, the $s^{\prime}$ contributions start from the second order in similarity to those of $s$ which are easily included. (ii) A scalar triplet $\rho$ similar to $\phi^{\prime}$ must be imposed. The $\rho$ is also skip for the same reason as $s^{\prime}$, that is, its contribution is similar to that of $\phi^{\prime}$. Let us emphasis that our conclusions remain unchanged if $s^{\prime}$ and $\rho$ present.

The hierarchies in the VEVs were given in [23]:

$$
\lambda_{1}, \lambda_{2}<u_{1}, u_{2}<v, v^{\prime}, u, u^{\prime}<\omega, \Lambda_{1}, \Lambda_{2} .
$$

In the following, the two limits are often taken into account: (i) the lepton-number violating parameters tend to zero, that is, $\lambda_{1,2}, u_{1,2} \rightarrow 0$, and (ii) the large scales of $\mathrm{SU}(3)_{L}$ symmetry break down to that of the standard model approx infinity, that is, $\omega, \Lambda_{1,2} \rightarrow \infty$. Let us note also that $v, v^{\prime}, u$, and $u^{\prime}$ are in the electroweak scale as well as the large scales all conserving the lepton number.

\section{Gauge Bosons}

The covariant derivative of a general triplet $\Phi$ is given by

$$
\begin{aligned}
D_{\mu} & =\partial_{\mu}+i g T_{a} W_{a \mu}+i g_{X} T_{9} X B_{\mu} \\
& \equiv \partial_{\mu}-i D_{\mu}
\end{aligned}
$$

where the gauge fields $W_{a}$ and $B$ transform as the adjoint representations of $\mathrm{SU}(3)_{L}$ and $\mathrm{U}(1)_{X}$, respectively, and the corresponding gauge coupling constants $g$ and $g_{X}$. The $T_{9}=$ $\operatorname{diag}(1,1,1) / \sqrt{6}$ is chosen so that $\operatorname{Tr}\left(T_{a} T_{b}\right)=\delta_{a b} / 2$ with $a, b=1,2, \ldots, 9$. The neutral gauge bosons of the theory get masses from the triplet as follows:

$$
\mathcal{L}_{\text {mass }}^{\Phi}=\left(D_{\mu}^{H}\langle\Phi\rangle\right)^{+}\left(D^{H \mu}\langle\Phi\rangle\right),
$$

where the subscript $H$ denotes diagonal part of the covariant derivative:

$$
D_{\mu}^{H}=\partial_{\mu}+i g T_{3} W_{\mu}^{3}+i g T_{8} W_{\mu}^{8}+i g_{X} T_{9} X_{X} B_{\mu} .
$$


The covariant derivative for an antisextet with the VEV part is [30]

$$
D_{\mu}\left\langle s_{i}\right\rangle=-\frac{i g}{2}\left\{A_{\mu}^{a} \lambda_{a}^{*}\left\langle s_{i}\right\rangle+\left\langle s_{i}\right\rangle A_{\mu}^{a} \lambda_{a}^{* T}\right\}+i g_{X} T_{9} X B_{\mu}\langle s\rangle
$$

Let us denote the antisextet in term of the $\mathrm{SU}(3)_{L}$ indices by $\Gamma_{i j}$. Then, the mass Lagrangian due to the antisextet's contribution is given by

$$
\mathcal{L}_{\text {mass }}^{\Gamma}=\left(D_{\mu}^{H}\langle\Gamma\rangle_{i j}\right)^{+}\left(D^{H \mu}\langle\Gamma\rangle_{i j}\right)
$$

Let us denote the following combinations:

$$
\begin{aligned}
& W_{\mu}^{\prime \pm} \equiv \frac{W_{1 \mu} \mp i W_{2 \mu}}{\sqrt{2}}, \\
& Y_{\mu}^{\prime \mp} \equiv \frac{W_{6 \mu} \mp i W_{7 \mu}}{\sqrt{2}} \\
& X_{\mu}^{\prime 0} \equiv \frac{W_{4 \mu}-i W_{5 \mu}}{\sqrt{2}}
\end{aligned}
$$

having defined charges under the generators of the $S U(3)_{L}$ group. For the sake of convenience in further reading, we note that $W_{4}$ and $W_{5}$ are pure real and imaginary parts of $X_{\mu}^{\prime 0}$ and $X_{\mu}^{\prime}{ }^{*}$, respectively:

$$
\begin{aligned}
& W_{4 \mu}=\frac{1}{\sqrt{2}}\left(X_{\mu}^{\prime 0}+X_{\mu}^{\prime 0 *}\right) \\
& W_{5 \mu}=\frac{i}{\sqrt{2}}\left(X_{\mu}^{\prime 0}-X_{\mu}^{\prime 0 *}\right) .
\end{aligned}
$$

Then $p_{\mu}$ is rewritten in a convenient form:

$$
\frac{g}{2}\left(\begin{array}{ccc}
W_{3 \mu}+\frac{1}{\sqrt{3}} W_{8 \mu}+t \sqrt{\frac{2}{3}} X B_{\mu} & \sqrt{2} W_{\mu}^{\prime+} & \sqrt{2} X_{\mu}^{\prime 0} \\
\sqrt{2} W_{\mu}^{\prime-} & -W_{3 \mu}+\frac{1}{\sqrt{3}} W_{8 \mu}+t \sqrt{\frac{2}{3}} X B_{\mu} & \sqrt{2} Y_{\mu}^{\prime-} \\
\sqrt{2} X_{\mu}^{\prime 0 *} & \sqrt{2} Y_{\mu}^{\prime+} & -\frac{2}{\sqrt{3}} W_{8 \mu}+t \sqrt{\frac{2}{3}} X B_{\mu}
\end{array}\right)
$$

with $t \equiv g_{X} / g$. 
The covariant derivative acting on the antisextet VEV is given by

$$
\begin{gathered}
D_{\mu}\left\langle s_{i}\right\rangle_{11}=-i g\left(\lambda_{i} W_{3 \mu}+\lambda_{i} \frac{1}{\sqrt{3}} W_{8 \mu}-t \lambda_{i} \sqrt{\frac{2}{3}} \frac{1}{3} B_{\mu}+\sqrt{2} u_{i} X_{\mu}^{0}\right) \\
D_{\mu}\left\langle s_{i}\right\rangle_{12}=-\frac{i g}{\sqrt{2}}\left(\lambda_{i} W_{\mu}^{-}+u_{i} Y_{\mu}^{-}\right), \\
D_{\mu}\left\langle s_{i}\right\rangle_{13}=-\frac{i g}{2}\left(u_{i} W_{3 \mu}-u_{i} \frac{1}{\sqrt{3}} W_{8 \mu}-t u_{i} \sqrt{\frac{2}{3}} \frac{2}{3} B_{\mu}+\sqrt{2} \Lambda_{i} X_{\mu}^{0}+\sqrt{2} \lambda_{i} X_{\mu}^{0 *}\right) \\
D_{\mu}\left\langle s_{i}\right\rangle_{22}=0 \\
D_{\mu}\left\langle s_{i}\right\rangle_{23}=-\frac{i g}{\sqrt{2}}\left(u_{i} W_{\mu}^{-}+\Lambda_{i 3} Y_{\mu}^{-}\right) \\
\left(\begin{array}{l}
\left.2 \Lambda_{i} \frac{1}{\sqrt{3}} W_{8 \mu}+t \Lambda_{i} \sqrt{\frac{2}{3}} \frac{1}{3} B_{\mu}-\sqrt{2} u_{i} X_{\mu}^{* 0}\right) \\
D_{\mu}\left\langle s_{i}\right\rangle_{12}=D_{\mu}\left\langle s_{i}\right\rangle_{21} \\
D_{\mu}\left\langle s_{i}\right\rangle_{13}=D_{\mu}\left\langle s_{i}\right\rangle_{31} \\
D_{\mu}\left\langle s_{i}\right\rangle_{23}=D_{\mu}\left\langle s_{i}\right\rangle_{32} .
\end{array}\right.
\end{gathered}
$$

The masses of gauge bosons in this model are followed from

$$
\begin{aligned}
\mathcal{L}_{\text {mass }}^{\mathrm{GB}}= & \left(D_{\mu}\langle\phi\rangle\right)^{+} D^{\mu}\langle\phi\rangle+\left(D_{\mu}\left\langle\phi^{\prime}\right\rangle\right)^{+} D^{\mu}\left\langle\phi^{\prime}\right\rangle+\left(D_{\mu}\langle x\rangle\right)^{+} D^{\mu}\langle x\rangle \\
& +\left(D_{\mu}\langle\eta\rangle\right)^{+} D^{\mu}\langle\eta\rangle+\left(D_{\mu}\left\langle\eta^{\prime}\right\rangle\right)^{+} D^{\mu}\left\langle\eta^{\prime}\right\rangle+\operatorname{Tr}\left[\left(D_{\mu}\left\langle s_{1}\right\rangle\right)^{+} D^{\mu}\left\langle s_{1}\right\rangle\right] \\
& +\operatorname{Tr}\left[\left(D_{\mu}\left\langle s_{2}\right\rangle\right)^{+} D^{\mu}\left\langle s_{2}\right\rangle\right] .
\end{aligned}
$$

In the following, we notice that $\left\langle s_{1}\right\rangle=\left\langle s_{2}\right\rangle$; namely, $u_{1}=u_{2}, \lambda_{1}=\lambda_{2}$, and $\Lambda_{1}=\Lambda_{2}$ are taken into account.

From (3.10), the imaginary part $W_{5}$ is decoupled with mass given by

$$
M_{W_{5}}^{2}=\frac{g^{2}}{2}\left(16 u_{1}^{2}+4 \lambda_{1}^{2}-8 \Lambda_{1} \lambda_{1}+4 \Lambda_{1}^{2}+\omega^{2}+u^{2}+u^{\prime 2}\right) .
$$

In the limit $\lambda_{1}, u_{1} \rightarrow 0$,

$$
M_{W_{5}}^{2}=\frac{g^{2}}{2}\left(u^{2}+u^{\prime 2}+\omega^{2}+4 \Lambda_{1}^{2}\right)
$$


The charged gauge bosons $W$ and $Y$ mix via

$$
\mathcal{L}_{\text {mass }}^{\mathrm{CG}}=\frac{g^{2}}{4}\left(W_{\mu}^{-}, Y_{\mu}^{-}\right) M_{W Y}^{2}\left(W_{\mu}^{+}, Y_{\mu}^{+}\right)^{T}
$$

where

$$
M_{W Y}^{2}=2\left(\begin{array}{cc}
v^{2}+v^{\prime 2}+u^{2}+u^{\prime 2}+4 u_{1}^{2}+4 \lambda_{1}^{2} & 4\left(\Lambda_{1} u_{1}+\lambda_{1} u_{1}\right) \\
4\left(\Lambda_{1} u_{1}+\lambda_{1} u_{1}\right) & v^{2}+v^{\prime 2}+\omega^{2}+4 \Lambda_{1}^{2}+4 u_{1}^{2}
\end{array}\right)
$$

Diagonalizing this mass matrix, we get physical charged gauge bosons

$$
\begin{aligned}
& W_{\mu}^{-}=\cos \theta W_{\mu}^{\prime-}+\sin \theta Y_{\mu}^{\prime-}, \\
& Y_{\mu}^{-}=-\sin \theta W_{\mu}^{\prime-}+\cos \theta Y_{\mu}^{\prime-} .
\end{aligned}
$$

The mixing angle is given by

$$
\tan \theta=\frac{4\left(\Lambda_{1} u_{1}+\lambda_{1} u_{1}\right)}{\omega^{2}+4 \Lambda_{1}^{2}-u^{2}-u^{\prime 2}-4 \lambda_{1}^{2}} \sim \frac{u_{1}}{\Lambda_{1}},
$$

provided that $\omega^{2} \sim \Lambda_{1}^{2} \gg u^{2}, u^{\prime 2}, u_{1}^{2}, \lambda_{1}^{2}$. The mass eigenvalues are

$$
\begin{aligned}
& M_{W}^{2}=\frac{g^{2}}{4}\left\{v^{2}+v^{\prime 2}+2 u^{2}+2 u^{\prime 2}+\omega^{2}+4 \lambda_{1}^{2}+4 \Lambda_{1}^{2}\right. \\
&- {\left[\left(v^{2}+v^{\prime 2}-\omega^{2}\right)^{2}+16 \lambda_{1}^{4}+16 \Lambda_{1}^{4}+128 \lambda_{1} \Lambda_{1} u_{1}^{2}\right.} \\
&\left.\left.+8 \Lambda_{1}^{2}\left(\omega^{2}-v^{2}-v^{\prime 2}+8 u_{1}^{2}\right)-8 \lambda_{1}^{2}\left(4 \Lambda_{1}^{2} \omega^{2}-v^{2}-v^{\prime 2}-8 u_{1}^{2}\right)\right]^{1 / 2}\right\} \\
& M_{Y}^{2}=\frac{g^{2}}{4}\left\{v^{2}+v^{\prime 2}+2 u^{2}+2 u^{\prime 2}+\omega^{2}+4 \lambda_{1}^{2}+4 \Lambda_{1}^{2}\right. \\
&+ {\left[\left(v^{2}+v^{\prime 2}-\omega^{2}\right)^{2}+16 \lambda_{1}^{4}+16 \Lambda_{1}^{4}+128 \lambda_{1} \Lambda_{1} u_{1}^{2}\right.} \\
&\left.\left.+8 \Lambda_{1}^{2}\left(\omega^{2}-v^{2}-v^{\prime 2}+8 u_{1}^{2}\right)-8 \lambda_{1}^{2}\left(4 \Lambda_{1}^{2} \omega^{2}-v^{2}-v^{\prime 2}-8 u_{1}^{2}\right)\right]^{1 / 2}\right\} .
\end{aligned}
$$

Note that, in the limit $\lambda_{1}, u_{1} \rightarrow 0$, the mixing angle tends to zero and the mass eigenvalues are

$$
\begin{aligned}
& M_{W}^{2}=\frac{g^{2}}{2}\left(v^{2}+v^{\prime 2}+u^{2}+u^{\prime 2}\right), \\
& M_{Y}^{2}=\frac{g^{2}}{2}\left(v^{\prime 2}+v^{2}+\omega^{2}+4 \Lambda_{1}^{2}\right) .
\end{aligned}
$$


There is a mixing among the neutral gauge bosons $W_{3}, W_{8}, B$, and $W_{4}$. The mass Lagrangian in this case has the form

$$
\begin{aligned}
\mathcal{L}_{\text {mass }}^{\mathrm{NG}} & =\frac{1}{2} V^{T} M^{2} V, \\
V^{T} & \equiv\left(W_{3}, W_{8}, B, W_{4}\right) .
\end{aligned}
$$

In the basis of these elements, the mass matrix is given by

$$
M^{2}=\frac{g^{2}}{4}\left(\begin{array}{llll}
M_{11}^{2} & M_{12}^{2} & M_{13}^{2} & M_{14}^{2} \\
M_{12}^{2} & M_{22}^{2} & M_{23}^{2} & M_{24}^{2} \\
M_{13}^{2} & M_{23}^{2} & M_{33}^{2} & M_{34}^{2} \\
M_{14}^{2} & M_{24}^{2} & M_{34}^{2} & M_{44}^{2}
\end{array}\right)
$$

where

$$
\begin{gathered}
M_{11}^{2}=2\left(4 u_{1}^{2}+8 \lambda_{1}^{2}+u^{2}+u^{\prime 2}+v^{2}+v^{\prime 2}\right), \\
M_{22}^{2}=\frac{2}{3}\left(4 u_{1}^{2}+8 \lambda_{1}^{2}+u^{2}+u^{\prime 2}+32 \Lambda_{1}^{2}+v^{2}+v^{\prime 2}+4 \omega^{2}\right), \\
M_{33}^{2}=\frac{4 t^{2}}{27}\left(16 u_{1}^{2}+8 \lambda_{1}^{2}+u^{2}+u^{\prime 2}+8 \Lambda_{1}^{2}+4 v^{2}+4 v^{\prime 2}+\omega^{2}\right), \\
M_{44}^{2}=2\left(\omega^{2}+u^{2}+u^{\prime 2}+16 u_{1}^{2}+4 \Lambda_{1}^{2}+4 \lambda_{1}^{2}+8 \Lambda_{1} \lambda_{1}\right), \\
M_{12}^{2}=\frac{2}{\sqrt{3}}\left(-4 u_{1}^{2}+8 \lambda_{1}^{2}+u^{2}+u^{\prime 2}-v^{2}-v^{\prime 2}\right), \\
M_{13}^{2}=-\frac{2}{3} \sqrt{\frac{2}{3}} t\left(8 u_{1}^{2}+8 \lambda_{1}^{2}+u^{2}+u^{\prime 2}+2 v^{2}+2 v^{\prime 2}\right), \\
M_{14}^{2}=8\left(3 u_{1} \lambda_{1}+u_{1} \Lambda_{1}\right), \\
M_{23}^{2}=\frac{2 \sqrt{2}}{9} t\left(8 u_{1}^{2}+8 \lambda_{1}^{2}-u^{2}-u^{\prime 2}+16 \Lambda_{1}^{2}+2 v^{2}+2 v^{\prime 2}+2 \omega^{2}\right), \\
M_{24}^{2}=\frac{8}{\sqrt{3}}\left(u_{1} \lambda_{1}-5 u_{1} \Lambda_{1}\right), \\
M_{34}^{2}=-\frac{32}{3} \sqrt{\frac{2}{3}} t\left(u_{1} \lambda_{1}+u_{1} \Lambda_{1}\right) .
\end{gathered}
$$

This mass matrix contains one exact eigenvalue:

$$
M_{\gamma}^{2}=0
$$


The associated eigenvector is

$$
A_{\mu}=\frac{1}{\sqrt{18+4 t^{2}}}\left(\begin{array}{c}
\sqrt{3} t \\
-t \\
3 \sqrt{2} \\
0
\end{array}\right)
$$

Using continuation of the gauge coupling constant $g$ of the $\mathrm{SU}(3)_{L}$ at the spontaneous symmetry breaking point, we have [2-9]

$$
t=\frac{3 \sqrt{2} s_{W}}{\sqrt{3-4 s_{W}^{2}}}
$$

In order to diagonalize the mass matrix, we choose the base of $\left(A_{\mu}, Z_{\mu}, Z_{\mu}^{\prime}, W_{4 \mu}\right)$, with

$$
\begin{gathered}
Z_{\mu}=c_{W} W_{3 \mu}-s_{W}\left(-\frac{t_{W}}{\sqrt{3}} W_{8 \mu}+\sqrt{1-\frac{t_{W}^{2}}{3}} B_{\mu}\right), \\
Z_{\mu}^{\prime}=\sqrt{1-\frac{t_{W}^{2}}{3}} W_{8 \mu}+\frac{t_{W}}{\sqrt{3}} B_{\mu} .
\end{gathered}
$$

The new base is changed from the old by unitary matrix:

$$
U=\left(\begin{array}{cccc}
s_{W} & c_{W} & 0 & 0 \\
-\frac{c_{W} t_{W}}{\sqrt{3}} & \frac{s_{W} t_{W}}{\sqrt{3}} & \sqrt{1-\frac{t_{W}^{2}}{3}} & 0 \\
c_{W} \sqrt{1-\frac{t_{W}^{2}}{3}} & -s_{W} \sqrt{1-\frac{t_{W}^{2}}{3}} & \frac{t_{W}}{\sqrt{3}} & 0 \\
0 & 0 & 0 & 1
\end{array}\right) .
$$

In this basis, the mass matrix $M^{2}$ becomes

$$
M^{\prime 2}=U^{+} M^{2} U=\frac{g^{2}}{4}\left(\begin{array}{cccc}
0 & 0 & 0 & 0 \\
0 & M_{22}^{\prime 2} & M_{23}^{\prime 2} & M_{24}^{\prime 2} \\
0 & M_{23}^{\prime 2} & M_{33}^{\prime 2} & M_{34}^{\prime 2} \\
0 & M_{24}^{\prime 2} & M_{34}^{\prime 2} & M_{44}^{\prime 2}
\end{array}\right) .
$$


In the approximation $\lambda_{1}^{2}, u_{1}^{2} \ll \Lambda_{1}^{2}$, we have

$$
\begin{aligned}
& M_{22}^{\prime 2}=2\left(u^{2}+u^{\prime 2}+v^{2}+v^{\prime 2}\right) \frac{1}{c_{W}^{2}} \\
& M_{23}^{\prime 2}=\frac{-2\left[2 s_{W}^{2}\left(u^{2}+u^{\prime 2}\right)-\left(v^{2}+v^{\prime 2}\right)\right] \sqrt{\alpha_{0}}}{c_{W}^{2}}, \\
& M_{24}^{\prime 2}=\frac{8 u_{1} \Lambda_{1}}{c_{W}}, \\
& M_{33}^{\prime 2}=\frac{2\left(u^{2}+u^{\prime 2}\right)}{c_{W}^{2} \alpha_{0}}-\frac{2\left(v^{2}+v^{\prime 2}\right) \alpha_{0}}{c_{W}^{2}}+8 \omega^{2} c_{W}^{2} \alpha_{0}+64 \Lambda_{1}^{2} c_{W}^{2} \alpha_{0}, \\
& M_{34}^{\prime 2}=\frac{-8 x_{0} u_{1} \Lambda_{1}}{c_{W} \sqrt{\alpha_{0}}}, \\
& M_{44}^{\prime 2}=2\left(\omega^{2}+u^{2}+u^{\prime 2}+4 \Lambda_{1} \lambda_{1}+4 \Lambda_{1}^{2}\right)
\end{aligned}
$$

with

$$
\begin{aligned}
& x_{0}=\left(4 c_{W}^{2}+1\right), \\
& \alpha_{0}=\frac{1}{\left(4 c_{W}^{2}-1\right)} .
\end{aligned}
$$

It is noteworthy that in the limit $u_{1}=0$, the elements $M_{24}^{\prime}$ and $M_{34}^{\prime}$ (or equivalently $M_{14}$, $M_{24}, M_{34}$ in the old base) vanish. In this case, the mixing between $W_{4}$ and $Z, Z^{\prime}$ disappears. Three bosons gain masses via seesaw mechanism:

$$
\begin{gathered}
M_{Z}^{2}=M_{22}^{\prime 2}-\left(M^{\text {off }}\right)^{T}\left(M_{2 X 2}^{\prime 2}\right)^{-1} M^{\text {off }}, \\
M_{2 X 2}^{2} \approx M_{2 X 2}^{\prime 2}
\end{gathered}
$$

where

$$
\begin{aligned}
M^{\text {off }} & =\left(\begin{array}{l}
M_{23}^{\prime 2} \\
M_{24}^{\prime 2}
\end{array}\right), \\
M_{2 X 2}^{\prime 2} & =\left(\begin{array}{ll}
M_{33}^{\prime 2} & M_{34}^{\prime 2} \\
M_{34}^{\prime 2} & M_{44}^{\prime 2}
\end{array}\right) .
\end{aligned}
$$


We have then

$$
\begin{aligned}
M_{Z}^{2} & =\frac{g^{2}}{4}\left(M_{22}^{\prime 2}-\frac{\left(M_{23}^{\prime 2}\right)^{2} M_{44}^{\prime 2}-2 M_{23}^{\prime 2} M_{24}^{\prime 2} M_{34}^{\prime 2}+\left(M_{24}^{\prime 2}\right)^{2} M_{33}^{\prime 2}}{M_{33}^{\prime 2} M_{44}^{\prime 2}-\left(M_{34}^{\prime 2}\right)^{2}}\right) \\
& =\frac{g^{2}}{2 c_{W}^{2}}\left(u^{2}+u^{\prime 2}+v^{2}+v^{\prime 2}\right)-\Delta_{M_{22}^{2}}
\end{aligned}
$$

where

$$
\begin{aligned}
\Delta_{M_{22}^{2}} & =\frac{g^{2}}{\alpha_{0}^{2}} \frac{\left[32\left(2 x_{0} x_{1}+x_{3}\right) u_{1}^{2} \Lambda_{1}^{2}+x_{1}^{2} x_{2}\right]}{x_{2} x_{3}-32 x_{0}^{2} u_{1}^{2} \Lambda_{1}^{2}}, \\
x_{1} & =c_{2 W}\left(u^{2}+u^{\prime 2}\right)-\left(v^{2}+v^{\prime 2}\right), \\
x_{2} & =\left(\omega^{2}+u^{2}+u^{\prime 2}+4 \Lambda_{1} \lambda_{1}+4 \Lambda_{1}^{2}\right), \\
x_{3} & =c_{2 W}\left(u^{2}+u^{\prime 2}\right)+v^{2}+v^{\prime 2}+4 c_{W}^{4} \omega^{2}+32 c_{W}^{4} \Lambda_{1}^{2} .
\end{aligned}
$$

The $\rho$ parameter in the our model is given by

$$
\rho=1+\delta_{\text {tree }}+\delta_{\text {loop }}
$$

where $\delta_{\text {loop }}$ gets contribution from the oblique correction depending on the masses of top quark and standard model Higgs boson [1]. The tree level correction $\delta_{\text {tree }}$ describes the new physics as given by

$$
\delta_{\text {tree }}=\frac{M_{W}^{2}}{c_{W}^{2} M_{Z}^{2}}-1 \simeq \frac{c_{W}^{2} \Delta_{M_{22}^{2}}}{M_{W}^{2}} .
$$

It is noted that $\Delta_{M_{22}^{2}} \neq 0$ even if $\omega$ and $\Lambda_{1}$ go to infinity. This is because the 33 components of antisextets and the third components of scalar triplets can be integrated out. There leave the standard model scalar doublets and triplets (the submultiplets of the 3-3-1 model triplets and antisextets). Such standard model scalar triplets imply $\delta_{\text {tree }} \neq 0$ to be given by

$$
\delta_{\text {tree }} \simeq \frac{8 g^{2} c_{W}^{2}\left(4 c_{W}^{2}-1\right)^{2} u_{1}^{2}}{M_{W}^{2}} \neq 0 .
$$

The $\delta_{\text {tree }}$ parameter has already been given in [1] as $\rho_{0}-1$ from the global fit:

$$
\delta_{\text {tree }}=0.0008_{-0.0007}^{+0.0017}
$$


Hence

$$
0.8403 \mathrm{GeV}^{2} \leq \Delta_{M_{22}^{2}} \leq 21.0065 \mathrm{GeV}^{2},
$$

or

$$
0.239 \mathrm{GeV} \leq u_{1} \leq 1.197 \mathrm{GeV}
$$

where we have used $c_{W}^{2}=0.769$ and $M_{W}=80.384 \mathrm{GeV}$.

Diagonalizing the mass matrix $M_{2 \mathrm{X} 2}^{2}$, we get new gauge bosons:

$$
\begin{gathered}
Z_{\mu}^{\prime}=\cos \phi Z_{\mu}^{\prime \prime}+\sin \phi W_{4 \mu^{\prime}}^{\prime} \\
W_{4 \mu}=-\sin \phi Z_{\mu}^{\prime \prime}+\cos \phi W_{4 \mu^{\prime}}^{\prime} .
\end{gathered}
$$

The mixing angle is defined by

$$
\tan \phi=\frac{2 M_{34}^{\prime 2}}{M_{44}^{\prime 2}-M_{33}^{\prime 2}+\sqrt{\left(M_{44}^{\prime 2}-M_{33}^{\prime 2}\right)^{2}+4\left(M_{34}^{\prime 2}\right)^{2}}} .
$$

Substituting (3.28) into (3.41), we get

$$
\begin{aligned}
\tan \phi=-2 \sqrt{\alpha_{1}} u_{1} \Lambda_{1} & \left\{\alpha_{2}\left(u^{2}+u^{\prime 2}\right)+\alpha_{3}\left(v^{2}+v^{\prime 2}\right)-2 \alpha_{0} w^{2}+8 \lambda_{1} \Lambda_{1}-8 x_{0} \alpha_{0} \Lambda_{1}^{2}\right. \\
& \left.+\left[\left(\alpha_{2}\left(u^{2}+u^{\prime 2}\right)+\alpha_{3}\left(v^{2}+v^{\prime 2}\right)-2 \alpha_{0} w^{2}+8 \lambda_{1} \Lambda_{1}-8 x_{0} \alpha_{0} \Lambda_{1}^{2}\right)^{2}+4 \alpha_{1} u_{1}^{2} \Lambda_{1}^{2}\right]^{1 / 2}\right\}^{-1} \\
\simeq & \frac{\sqrt{4 c_{W}^{2}-1}}{c_{W}} \frac{u_{1}}{\Lambda_{1}},
\end{aligned}
$$

provided that $\omega^{2} \sim \Lambda_{1}^{2} \gg u^{2}, u^{\prime 2}, v^{2}, v^{\prime 2}, u_{1}^{2}, \lambda_{1}^{2}$, where

$$
\begin{aligned}
& \alpha_{1}=\frac{64 x_{0}^{2} \alpha_{0}}{c_{W}^{2}}, \\
& \alpha_{2}=\frac{-2+6 c_{W}^{2}}{c_{W}^{2}} \alpha_{0}, \\
& \alpha_{3}=\frac{-2}{c_{W}^{2}} \alpha_{0} .
\end{aligned}
$$


The physical mass eigenvalues are defined by

$$
\begin{aligned}
M_{Z^{\prime \prime}, W_{4 \mu}^{\prime}}^{2}= & \frac{g^{2}}{4} \frac{M_{44}^{\prime 2}+M_{33}^{\prime 2} \mp \sqrt{\left(M_{44}^{\prime 2}-M_{33}^{\prime 2}\right)^{2}+4\left(M_{34}^{\prime 2}\right)^{2}}}{2} \\
= & \frac{g^{2}}{8}\left(\alpha_{4}\left(u^{2}+u^{\prime 2}\right)-\alpha_{3}\left(v^{2}+v^{\prime 2}\right)+\alpha_{5} w^{2}+8 \lambda_{1} \Lambda_{1}+\alpha_{6} \Lambda_{1}^{2}\right. \\
& \pm \sqrt{\left.\left(\alpha_{2}\left(u^{2}+u^{\prime 2}\right)+\alpha_{3}\left(v^{2}+v^{\prime 2}\right)-2 \alpha_{0} w^{2}+8 \lambda_{1} \Lambda_{1}-8 x_{0} \alpha_{0} \Lambda_{1}^{2}\right)^{2}+4 \alpha_{1} u_{1}^{2} \Lambda_{1}^{2}\right)}
\end{aligned}
$$

with

$$
\begin{aligned}
& \alpha_{4}=\frac{2-10 c_{W}^{2}+16 c_{W}^{4}}{c_{W}^{2}} \alpha_{0}, \\
& \alpha_{5}=\left(16 c_{W}^{2}-2\right) \alpha_{0}, \\
& \alpha_{6}=8\left(12 c_{W}^{2}-1\right) \alpha_{0} .
\end{aligned}
$$

In the limit $\lambda_{1}, u_{1} \rightarrow 0$, we have

$$
\begin{gathered}
M_{Z^{\prime \prime}}^{2}=\frac{g^{2}\left[c_{2 W}^{2}\left(u^{2}+u^{\prime 2}\right)+v^{2}+v^{\prime 2}+4 c_{W}^{4} \omega^{2}+32 c_{W}^{4} \Lambda_{1}^{2}\right]}{2 c_{W}^{2}} \alpha_{0}, \\
M_{W^{\prime} 4}^{2}=\frac{g^{2}}{2}\left(u^{2}+u^{\prime 2}+\omega^{2}+4 \Lambda_{1}^{2}\right) .
\end{gathered}
$$

Thus the $W_{4}^{\prime}$ and $W_{5}$ components have the same mass. With this result, we should identify the combination of $W^{\prime}{ }_{4}$ and $W_{5}$

$$
\sqrt{2} X_{\mu}^{0}=W_{4 \mu}^{\prime}-i W_{5 \mu}
$$

as physical neutral non-Hermitian gauge boson. The subscript 0 denotes neutrality of gauge boson $X$. However, to get tribimaximal mixing, the previous limit is not valid [21, 22]. This means that neutrino tribimaximal mixing leads to the masses of $X^{0}$ and $X^{0 \dagger}$ to be different. Consequence of this fact is that there is CPT violation $[1,31]$ in the model under consideration. We will return to this problem in the future work.

In the limit $\omega^{2} \sim \Lambda_{1}^{2} \gg u^{2}, u^{\prime 2}, v^{2}, v^{\prime 2}, u_{1}^{2}, \lambda_{1}^{2}$ (or $\omega, \Lambda_{1} \rightarrow \infty$ ), the mixings between the charged gauge bosons $W-Y$ and the neutral ones $W_{4}-Z^{\prime}$ are in the same order since from (3.16) and (3.42) they are proportional to $u_{1} / \Lambda_{1}$. In addition, from (3.46), $M_{Z^{\prime \prime}}^{2} \simeq 2 g^{2}\left(\omega^{2}+\right.$ $\left.16 c_{W}^{2} \Lambda_{1}^{1}\right)$ is bigger than $M_{W_{4}}^{2} \simeq\left(g^{2} / 2\right)\left(\omega^{2}+4 \Lambda_{1}^{2}\right)$ (or $\left.M_{X^{0}}^{2}\right)$. It is also verified that $\left|M_{Y}^{2}-M_{X^{0}}^{2}\right|<$ $M_{W}^{2}$. In that limit, the masses of $X^{0}$ and $Y$ degenerate. 
Note that the formulas for masses and mixing of gauge bosons previously presented, are common for the 3-3-1 models with more complicated Higgs sector such as with $A_{4}$ or $S_{4}$ discrete symmetries.

\section{Charged Currents}

The interaction among fermions with gauge bosons arises from part

$$
i \bar{\psi} \gamma_{\mu} D^{\mu} \psi=\text { kinematic terms }+H^{\mathrm{CC}}+H^{\mathrm{NC}} .
$$

Similarly in the economical 3-3-1 model, despite neutrality, the gauge bosons $X^{0}$ and $X^{0 *}$ belong to this section by their nature. Because of the mixing among the SM $W$ boson and the charged bilepton $Y$ as well as among $\left(X^{0}+X^{0 *}\right)$ with $\left(W_{3}, W_{8}, B\right)$, the new terms exist the same as the economical 3-3-1 model $[25,26]$ :

$$
H^{\mathrm{CC}}=\frac{g}{\sqrt{2}}\left(J_{W}^{\mu-} W_{\mu}^{+}+J_{Y}^{\mu-} Y_{\mu}^{+}+J_{X}^{\mu 0 *} X_{\mu}^{0}+\text { H.c. }\right) \text {, }
$$

where

$$
\begin{aligned}
J_{W}^{\mu-}= & c_{\theta}\left(\bar{v}_{i L} \gamma^{\mu} e_{i L}+\bar{u}_{i L} \gamma^{\mu} d_{i L}\right)+s_{\theta}\left(\bar{v}_{i L}^{c} \gamma^{\mu} e_{i L}+\bar{U}_{L} \gamma^{\mu} d_{1 L}+\bar{u}_{\alpha L} \gamma^{\mu} D_{\alpha L}\right), \\
J_{Y}^{\mu-}= & c_{\theta}\left(\bar{v}_{i L}^{c} \gamma^{\mu} e_{i L}+\bar{U}_{L} \gamma^{\mu} d_{1 L}+\bar{u}_{\alpha L} \gamma^{\mu} D_{\alpha L}\right)-s_{\theta}\left(\bar{v}_{i L} \gamma^{\mu} e_{i L}+\bar{u}_{i L} \gamma^{\mu} d_{i L}\right), \\
J_{X}^{\mu 0 *}= & \left(1-t_{2 \theta}^{2}\right)\left(\bar{v}_{i L} \gamma^{\mu} v_{i L}^{c}+\bar{u}_{1 L} \gamma^{\mu} U_{L}-\bar{D}_{\alpha L} \gamma^{\mu} d_{\alpha L}\right)-t_{2 \theta}^{2}\left(\bar{v}_{i L}^{c} \gamma^{\mu} v_{i L}+\bar{U}_{L} \gamma^{\mu} u_{1 L}-\bar{d}_{\alpha L} \gamma^{\mu} D_{\alpha L}\right) \\
& +\frac{t_{2 \theta}}{\sqrt{1+4 t_{2 \theta}^{2}}}\left(\bar{v}_{i} \gamma^{\mu} v_{i}+\bar{u}_{1 L} \gamma^{\mu} u_{1 L}-\bar{U}_{L} \gamma^{\mu} U_{L}-\bar{d}_{\alpha L} \gamma^{\mu} d_{\alpha L}+\bar{D}_{\alpha L} \gamma^{\mu} D_{\alpha L}\right) .
\end{aligned}
$$

All aforementioned interactions are lepton-number violating and weak (proportional to $\sin \theta$ or its square $\sin ^{2} \theta$ ). However, these couplings lead to lepton-number violations only in the neutrino sector.

Let us consider some constraints on the parameters of the model; one of the ways to do that is the consideration for $W$ decay. In our model, the $W$ boson has the following normal main decay modes:

$$
\begin{aligned}
& W^{-} \longrightarrow l \tilde{v}_{l} \quad(l=e, \mu, \tau), \\
& \searrow u^{c} d, u^{c} s, u^{c} b, \quad(u \rightarrow c),
\end{aligned}
$$

which are the same as in the SM and in the 331 with right-handed neutrinos. Beside the aforementioned modes, there are additional ones which are lepton-number violating $(\Delta L=2)$ the model's specific feature:

$$
W^{-} \longrightarrow l v_{l}(l=e, \mu, \tau)
$$




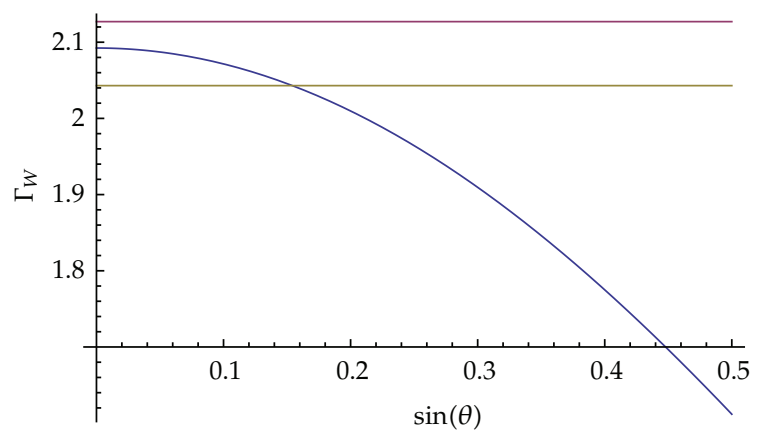

Figure 1: The total decay width of $W[\mathrm{GeV}]$ is depicted as a function of $\sin (\theta)$. The two horizontal lines are the upper and lower limits, respectively.

The interaction that provides these modes is as follows:

$$
\mathscr{L}=\frac{g}{\sqrt{2}} s_{\theta} \bar{v}_{i L}^{c} r^{\mu} e_{i L}
$$

where $v_{L}^{c} \equiv\left(N_{R}\right)^{c}$ is related to $v_{L}$ via the seesaw mechanism given by $v_{L}^{c}=M_{D} M_{R}^{-1} v_{L}$. Here $M_{R}$ and $M_{D}$ are right-handed Majorana and Dirac mass matrices (due to the contribution of $s)$, respectively, which can be derived from the Yukawa Lagrangian above to yield $M_{D} M_{R}^{-1}=$ $\left(u_{1} / \Lambda_{1}\right) \operatorname{diag}(1,1,1)$. On the other hand, from (3.16) we have $s_{\theta} \simeq \tan \theta \simeq u_{1} / \Lambda_{1}$ if $\Lambda_{1}$ is largest among the VEVs. It is therefore that $v_{L}^{c} \simeq s_{\theta} v_{L}$ and

$$
\mathscr{L}=\frac{g}{\sqrt{2}} s_{\theta}^{2} \bar{v}_{i L} \gamma^{\mu} e_{i L}
$$

The total decay width of $W$ is given by $[25,26]$

$$
\Gamma_{W}=1.039 \frac{\alpha M_{W}}{2 s_{W}^{2}}\left(1-s_{\theta}^{2}\right)+\frac{\alpha M_{W}}{4 s_{W}^{2}}\left(1-s_{\theta}^{2}\right)+\frac{\alpha M_{W}}{4 s_{W}^{2}} s_{\theta^{\prime}}^{4}
$$

where the first term is due to the quark productions (with $\alpha_{s}=0.1184$ chosen for the QCD radiative corrections), the second term comes from the normal modes with leptons, and the last one is for the unnormal modes. Let us choose $\alpha\left(M_{Z}\right)=1 / 128, M_{W}=80.399 \mathrm{GeV}$, and $\Gamma_{W}^{\mathrm{ext}}=2.085 \pm 0.042 \mathrm{GeV}$ [1]. The total decay width is plotted in Figure 1. From the figure, we get an upper limit on the $\sin \theta$ in the model:

$$
\sin \theta \leq 0.15
$$

which is bigger than that given in $[25,26]$.

There are lepton number violating interactions in the neutral Gauge boson sector, we refer interested reader to $[25,26]$. 


\section{Conclusions}

In this paper, we have investigated Gauge boson sector: their mixing and masses. The vacuum expectation values $u_{i}$ and $\lambda_{i}$ are a source of lepton-number violations and a reason for the mixing between the charged Gauge bosons-the standard model $W$ and the singly-charged bilepton Gauge bosons, as well as between neutral non-Hermitian $X^{0}$ and neutral Gauge bosons: the photon, the $Z$, and the new exotic $Z^{\prime}$. The interesting new physics compared with 3-3-1 models is the neutrino physics. Due to lepton-number violating couplings, we have many interesting consequences. We have shown that the neutrino tribimaximal mixing leads to the CPT violation. This feature will be considered in the future publication.

\section{Acknowledgment}

This work was supported in part by the National Foundation for Science and Technology Development (NAFOSTED) of Vietnam under Grant no. 103.01-2011.63.

\section{References}

[1] K. Nakamura, "Review of particle physics," Journal of Physics G, vol. 37, Article ID 075021, 2010.

[2] F. Pisano and V. Pleitez, "SU(3)U(1) model for electroweak interactions," Physical Review D, vol. 46, no. 1, pp. 410-417, 1992.

[3] P. H. Frampton, "Chiral dilepton model and the flavor question," Physical Review Letters, vol. 69, no. 20, pp. 2889-2891, 1992.

[4] R. Foot, O. F. Hernandez, F. Pisano, and V. Pleitez, "Lepton masses in an SU(3) $\otimes \otimes \mathrm{U}(1)_{N}$ Gauge model," Physical Review D, vol. 47, p. 4158, 1993.

[5] M. Singer, J. W. F. Valle, and J. Schechter, "Canonical neutral-current predictions from the weakelectromagnetic gauge group SU(3) × U(1)," Physical Review D, vol. 22, no. 3, pp. 738-743, 1980 .

[6] R. Foot, H. N. Long, and T. A. Tran, "SU(3) $)_{L} \mathrm{U}(1)_{N}$ and $\mathrm{SU}(4)_{L} \mathrm{U}(1)_{N}$ gauge models with right-handed neutrinos," Physical Review D, vol. 50, no. 1, pp. R34-R38, 1994.

[7] J. C. Montero, F. Pisano, and V. Pleitez, "Neutral currents and GIM mechanism in SU(3) ${ }_{L} \otimes U(1)_{N}$ model for electroweak interactions," Physical Review, vol. 47, pp. 2918-2929, 1993.

[8] H. N. Long, "SU(3) $L_{L} \otimes \mathrm{U}(1)_{N}$ model for right-handed neutrino neutral currents," Physical Review D, vol. 54, no. 7, pp. 4691-4693, 1996.

[9] H. N. Long, "SU(3) ${ }_{C} \otimes \mathrm{SU}(3)_{L} \otimes \mathrm{U}(1)_{N}$ model with right-handed neutrinos," Physical Review D, vol. 53, no. 1, pp. 437-445, 1996.

[10] H. N. Long and V. T. Van, "Quark family discrimination and flavor changing neutral currents in the $\mathrm{SU}(3)_{C} \otimes \mathrm{SU}(3)_{L} \otimes \mathrm{U}(1)$ model with right-handed neutrinos," Journal of Physics G, vol. 25, pp. 2319 2324, 1999.

[11] P. V. Dong and H. N. Long, "Neutrino masses and lepton flavor violation in the 3-3-1 model with right-handed neutrinos," Physical Review D, vol. 77, no. 5, Article ID 057302, 2008.

[12] F. Pisano, "A simple solution for the flavor question," Modern Physics Letters A, vol. 11, no. 32-33, pp. 2639-2647, 1996.

[13] A. Doff and F. Pisano, "Charge quantization in the largest leptoquark-bilepton chiral electroweak scheme," Modern Physics Letters A, vol. 14, no. 17, pp. 1133-1142, 1999.

[14] C. A. D. S. Pires and O. P. Ravinez, "Electric charge quantization in a chiral bilepton gauge model," Physical Review D, vol. 58, no. 3, Article ID 035008, 5 pages, 1998.

[15] C. A. D. S. Pires, "Remark on the vector-like nature of the electromagnetism and the electric charge quantization," Physical Review D, vol. 60, Article ID 075013, 1999.

[16] P. V. Dong and H. N. Long, "Electric charge quantization in $\mathrm{SU}(3)_{C} \otimes \mathrm{SU}(3)_{L} \otimes \mathrm{U}(1)_{X}$ models," International Journal of Modern Physics, vol. 21, pp. 6677-6692, 2000.

[17] P. F. Harrison, D. H. Perkins, and W. G. Scott, "Tri-bimaximal Lepton mixing and the neutrino oscillation data," Physics Letters B, vol. 530, p. 167, 2002. 
[18] Z.-Z. Xing, "Nearly tri-bimaximal neutrino mixing and CP violation," Physics Letters, Section B, vol. 533, no. 1-2, pp. 85-93, 2002.

[19] X. -G. He and A. Zee, "Some simple mixing and mass matrices for neutrinos," Physics Letters, Section $B$, vol. 560, no. 1-2, pp. 87-90, 2003.

[20] X. -G. He and A. Zee, “Neutrino masses with a "zero sum" condition: Mv1 + mv2 +mv3 = 0," Physical Review D, vol. 68, no. 3, Article ID 037302, 2003.

[21] P. V. Dong, L. T. Hue, H. N. Long, and D. V. Soa, "The 3-3-1 model with A4 flavor symmetry," Physical Review D, vol. 81, no. 5, Article ID 053004, 2010.

[22] P. V. Dong, H. N. Long, D. V. Soa, and V. V. Vien, “The 3-3-1 model with S4 flavor symmetry," European Physical Journal C, vol. 71, no. 2, Article ID 1544, pp. 1-11, 2011.

[23] P. V. Dong, H. N. Long, C. H. Nam, and V. V. Vien, "The $S_{3}$ flavor symmetry in 3-3-1 model," http:/ /arxiv.org/abs/1111.6360, to be published in Physical Review D.

[24] W. A. Ponce, Y. Giraldo, and L. A. Sánchez, "Minimal scalar sector of 3-3-1 models without exotic electric charges," Physical Review D, vol. 67, no. 7, Article ID 075001, 2003.

[25] P. V. Dong, H. N. Long, D. T. Nhung, and D. V. Soa, “SU(3) $C$ SU(3) $U(1)_{X}$ model with two Higgs triplets," Physical Review D, vol. 73, no. 3, Article ID 035004, pp. 1-11, 2006.

[26] P. V. Dong and H. N. Long, "The Economical SU(3) $C \otimes \mathrm{SU}(3)_{L} \otimes \mathrm{U}(1)_{X}$ Model," Advances in High Energy Physics, vol. 2008, Article ID 739492, 74 pages, 2008.

[27] E. Ma, "Non-Abelian discrete family symmetries of Leptons and quarks," http://arxiv.org/abs/hep$\mathrm{ph} / 0409075$.

[28] H. Ishimori, T. Kobayashi, H. Ohki, Y. Shimizu, H. Okada, and M. Tanimoto, “Non-Abelian discrete symmetries in particle physics," Progress of Theoretical Physics, no. 183, supplement, pp. 1-163, 2010.

[29] D. Chang and H. N. Long, "Interesting radiative patterns of neutrino mass in an $\mathrm{SU}(3)_{C} \mathrm{SU}(3)_{L} \mathrm{U}(1)_{X}$ model with right-handed neutrinos," Physical Review D, vol. 73, no. 5, Article ID 053006, pp. 1-17, 2006.

[30] P. V. Dong and H. N. Long, "U(1)Q invariance and $S U(3)_{C} \otimes S U(3)_{L} \otimes U(1)_{X}$ models with beta arbitrary," European Physical Journal C, vol. 42, no. 3, pp. 325-329, 2005.

[31] S. Hollenberg and P. B. Pal, "CPT-violating effects in muon decay," Physics Letters, Section B, vol. 701, no. 1, pp. 89-92, 2011. 

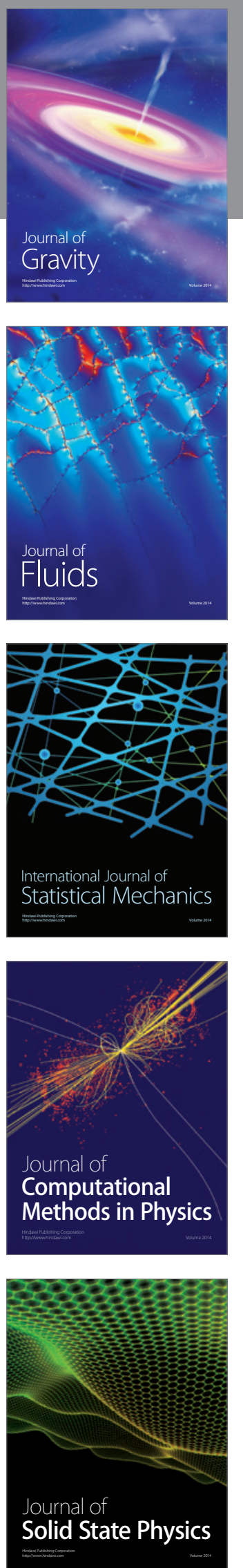

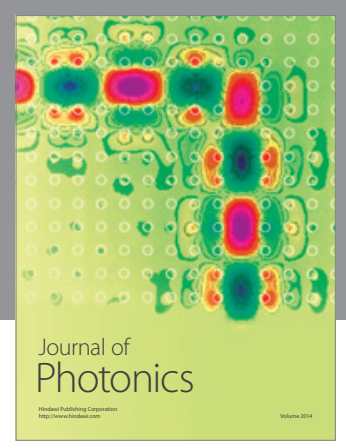

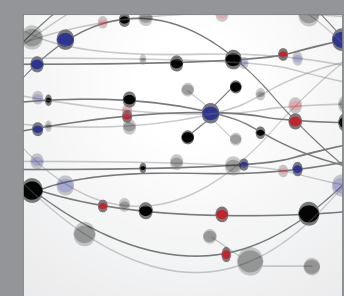

The Scientific World Journal
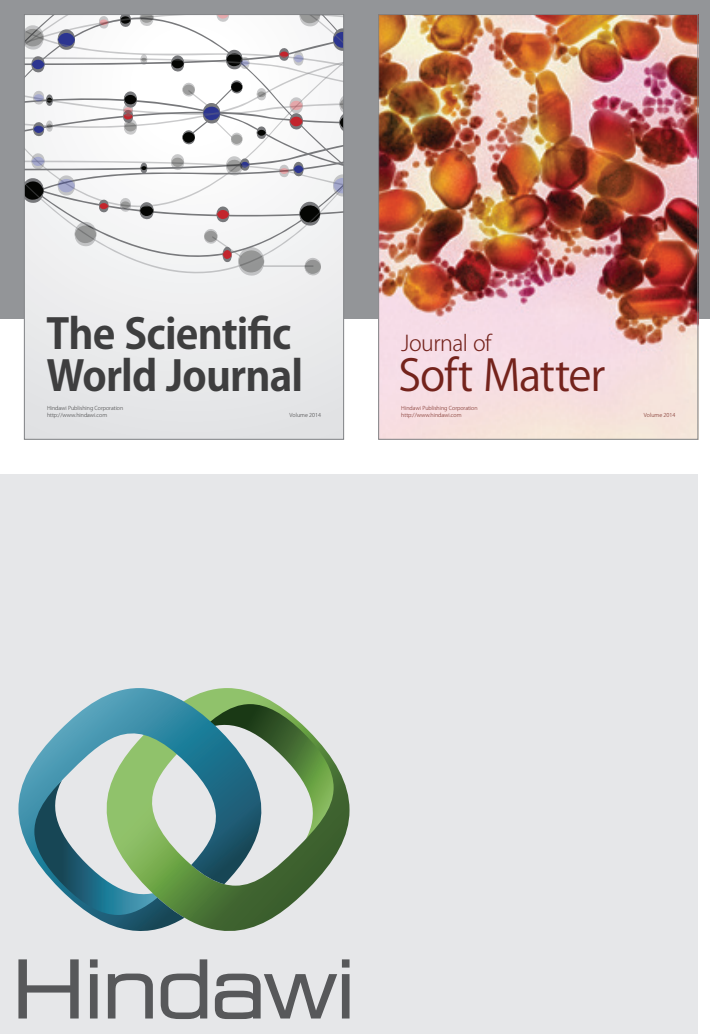

Submit your manuscripts at

http://www.hindawi.com
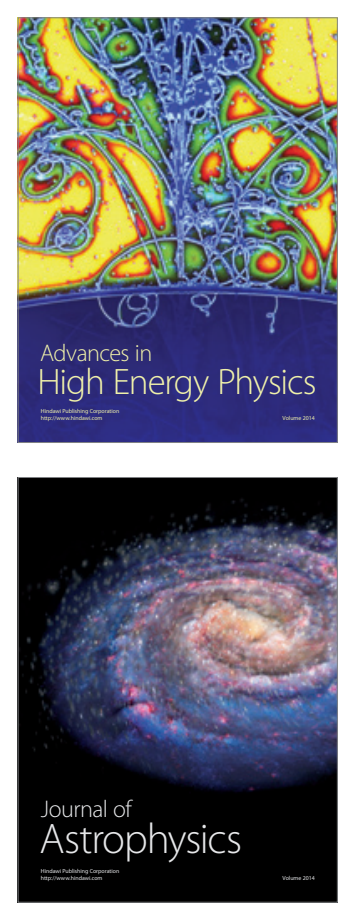
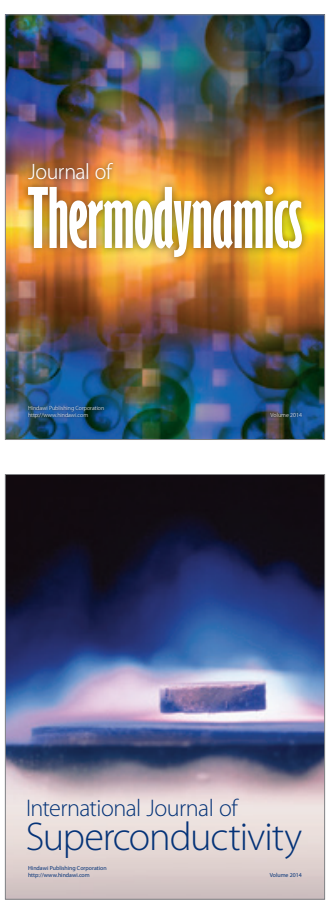
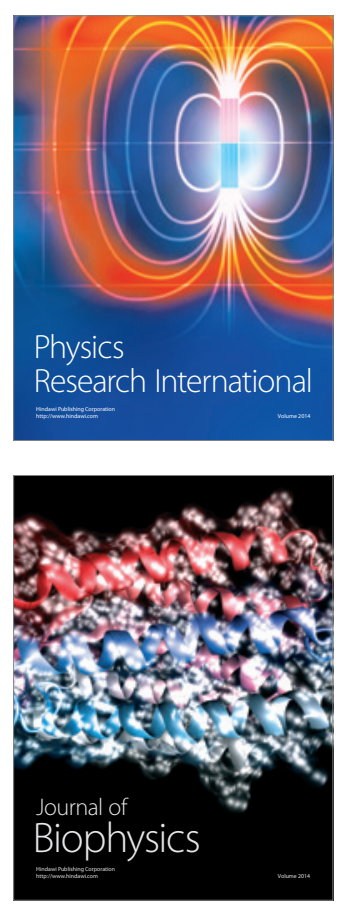
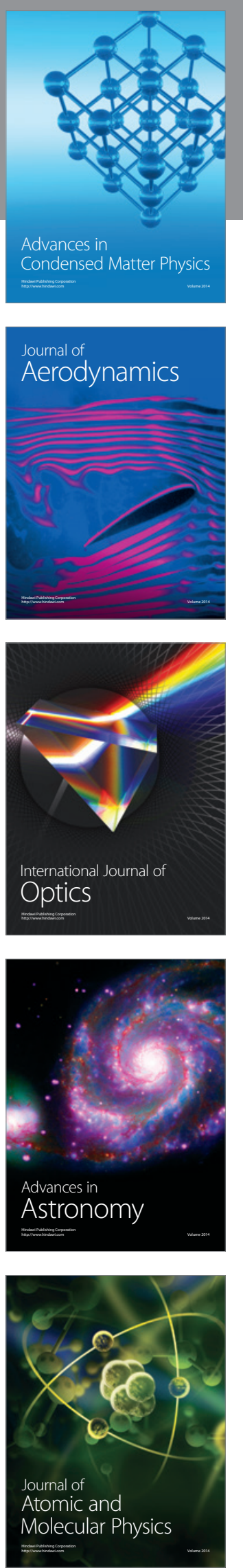